\title{
Photodynamic therapy for acne vulgaris: a randomized, controlled, split-face clinical trial of topical aminolevulinic acid and pulsed dye laser therapy
}

\author{
Jeffrey S Orringer, MD, Dana L Sachs, MD, Evans Bailey, MD, PhD, Sewon Kang, MD, \\ Ted Hamilton, MS, \& John J Voorhees, MD, FRCP \\ Department of Dermatology, University of Michigan Medical School, Ann Arbor, Michigan, USA
}

\begin{abstract}
Summary
Background There remains the need for more effective therapeutic options to treat acne vulgaris. Interest in light-based acne treatments has increased, but few randomized, controlled clinical trials assessing the value of photodynamic therapy (PDT) for acne have been reported.

Aims We sought to examine the efficacy of PDT using 5-aminolevulinic acid (ALA) and pulsed dye laser therapy in the treatment of acne.

Patients/methods We conducted a randomized, controlled, split-face, single-blind clinical trial of 44 patients with facial acne. Patients were randomized to receive three pulsed dye laser treatments to one side of the face after a 60-90 min ALA application time, while the contralateral side remained untreated and served as a control. Serial blinded lesion counts and global acne severity ratings were performed.

Results Global acne severity ratings improved bilaterally with the improvement noted to be statistically significantly greater in treated skin than in untreated skin. Erythematous macules (remnants of previously active inflammatory lesions) decreased in number in treated skin when compared with control skin and there was a transient but significant decrease in inflammatory papules in treated skin when compared with untreated skin. There were no other statistically significant differences between treated and untreated sides of the face in terms of counts of any subtype of acne lesion. Thirty percent of patients were deemed responders to this treatment with respect to improvement in their inflammatory lesion counts, while only $7 \%$ of patients responded in terms of noninflammatory lesion counts.

Conclusions PDT with the treatment regimen employed here may be beneficial for a subgroup of patients with inflammatory acne.
\end{abstract}

Keywords: photodynamic therapy, pulsed dye laser, 5-aminolevulinic acid, acne vulgaris

Correspondence: Jeffrey S Orringer, Department of Dermatology, University of Michigan Medical School, 1500 E. Medical Center Drive, 1910 Taubman Center, Ann Arbor, Ml 48109-0314, USA. E-mail: jorringe@umich.edu

Accepted for publication November 28, 2009

\section{Introduction}

Acne vulgaris is among the most common cutaneous disorders and it is associated with the potential for significantly disfiguring scarring and psychosocial morbidity. ${ }^{1-3}$ Although many traditional oral and topical medical agents have been demonstrated to be effective in the treatment of acne, the prevalence of the disease and 
its frequently resistant nature make the development of alternative therapies highly desirable.

Light-based approaches to the treatment of acne date back many years, but recently there has been renewed interest in this area. A number of lasers and other light sources have been assessed as possible treatments for acne, and several authors have reported promising initial results. ${ }^{4-9}$ However, many of the studies reported to date have inherent design-based limitations such as a lack of a control group, patients being allowed to remain on other anti-acne therapies during the course of the studies, and low patient numbers that make statistical analysis of the work challenging.

Pulsed dye laser therapy has been reported by some investigators to be efficacious in the treatment of acne vulgaris, while this has been refuted by other researchers. ${ }^{10,11}$ In an attempt to enhance the efficacy of these and other visible light wavelength devices in the treatment of acne, several investigators have employed a topical photosensitizer prior to laser/light therapy. ${ }^{12-15}$ One such treatment approach that has been advocated is the application of topical 20\% 5-aminolevulinic acid used in conjunction with pulsed dye laser therapy. ${ }^{16}$ We sought to objectively assess photodynamic therapy (PDT) using this popular treatment protocol in a randomized, blinded, split-face controlled clinical trial.

\section{Methods}

This study was approved by the institutional review board of the University of Michigan Medical School, and informed consent was obtained from all study subjects and parental consent was obtained for patients under 18 years of age. Patient recruitment occurred between January 2005 and January 2008, and the study was completed in July 2008. Inclusion criteria included an age of 13 years or older for patients of either gender and of any racial/ethnic group. Patients were required to have clinically evident facial acne at the time of study entry and were felt to be in generally good health and willing and able to comply with the requirements of the protocol. Potential patients were excluded from participation for the use of an oral retinoid within 1 year, systemic acne therapies (such as oral antibiotics) within 4 weeks, or topical acne therapies including over-the-counter products or prescription medications (retinoids, antibiotics) within 2 weeks of entry into the study. Patients were also excluded for a history of having undergone microdermabrasion or superficial chemical peels at the sites to be treated within 2 months of entry into the study or dermabrasion or laser resurfacing at the sites to be treated at any time. Noncompliant patients, those with a significant medical history or concurrent illness/condition that the investigators felt was not safe for study participation, and pregnant or nursing patients were also excluded. In addition, excluded were patients with a history of frequent herpes simplex infections of the face or with clinical evidence of active herpes simplex infection, those with a history of keloid scar formation, patients with a known allergy or hypersensitivity to topical photosensitizing agents, and those with known photosensitivity disorders.

The study was a randomized, controlled, split-face, single-blind clinical trial. Patients were randomized to receive topical photosensitizer applications followed by pulsed dye laser therapy to one side of the face and the contralateral side of the face remained untreated, thus serving as an internal control. A randomized code determined which side of each patient's face was to be treated. Patients received a total of up to three treatment sessions spaced at approximately 2-week intervals. Acetone scrubs were performed prior to the application of 20\% 5-aminolevulinic acid (Levulan, DUSA Pharmaceuticals, Inc., Willmington, MA, USA), which was left in place for 60-90 min prior to each treatment. Laser therapy was performed using a pulsed dye laser (VBeam, Candela Coroporation, Wayland, MA, USA) using the following treatment parameters: 10-mm spot size, 10-ms pulse duration, and 6.5$7.5 \mathrm{~J} / \mathrm{cm}^{2}$. (Patients with Fitzpatrick types I-IV skin were treated at $7.5 \mathrm{~J} / \mathrm{cm}^{2}$, while those of darker skin types were treated at lower fluences because of their relatively increased risk of postinflammatory hyperpigmentation.) A single pass of minimally overlapping pulses was applied to one side of the face. Treatment parameters were nonpurpuric and pulse stacking was not performed. The treating physicians (JS Orringer and DL Sachs) were not involved in clinical evaluations of the patients. Strict light precautions were required of patients for at least $48 \mathrm{~h}$ after each treatment session. Clinical evaluations including live lesion counts and global grading using the modified Leeds acne severity scale were performed at baseline and then every 2 weeks for a total of up to 16 weeks. ${ }^{17}$ Counts of papules, pustules, cysts, open comedones, closed comedones, and erythematous macules were performed. The latter category was intended to account for inactive, resolving previously inflammatory lesions. The Leeds scale is a 12-point ordinal photonumeric global acne severity scale where a rating of 1 denotes the mildest acne and a rating of 12 represents the most severe. 


\section{Statistical methods}

For all endpoints, the change from baseline for the treated side of the face was compared with the change from baseline on the untreated side in all patients. The data were analyzed with the Student's $t$-test for paired data. All $P$ values are two-tailed. Descriptive statistics are presented as means and their respective standard errors. The data were analyzed with sAs analytic software (SAS Institute Inc., Cary, NC, USA).

Mixed model fitting the lesion count with covariates age, gender, race, severity rating, continuous time, treatment, and time-treatment interaction, with random intercept and time was used to obtain predicted values where data were missing. Respondents were classified as individuals who had at least $25 \%$ fewer lesions at week 6 on the treated side when compared with the untreated side, and also had at least $25 \%$ fewer lesions on the treated side at week 6 compared with baseline lesion counts. Fisher's exact test was performed to check the association between the two criteria. Logistic regression was used to check if age, gender, race, treatment, or total number of lesions were predictive of responder status. The two-sided paired $t$-test was performed to compare responders and nonresponders on baseline lesion counts by type. Analysis was performed for responder classification based on total lesion counts, inflammatory lesions alone (defined by the sum of papules, pustules, and cysts), and noninflammatory lesions alone (defined by the sum of closed and open comedones).

\section{Results}

A total of 99 patients were screened for study participation and 44 patients were found to meet eligibility requirements and were randomized for inclusion in the study (Fig. 1).

Among enrolled patients, 14 were male, 30 were female, and patients were of a mean age of 25 years (range 15-50 years). Twenty-four patients were randomized to receive treatments to the left side of the face and 20 to the right side. A total of 29 patients completed the entire 16-week long study and 33 presented for at least one follow-up evaluation visit after their series of treatments.

On week 10 of the study, there was a statistically significant decrease from baseline in mean inflammatory papule counts in treated skin $(-4.63)$ when compared with untreated skin $(-0.13), P=0.01$, but this effect was transient with no significant benefit in papule counts noted at the conclusion of the study on week 16 .

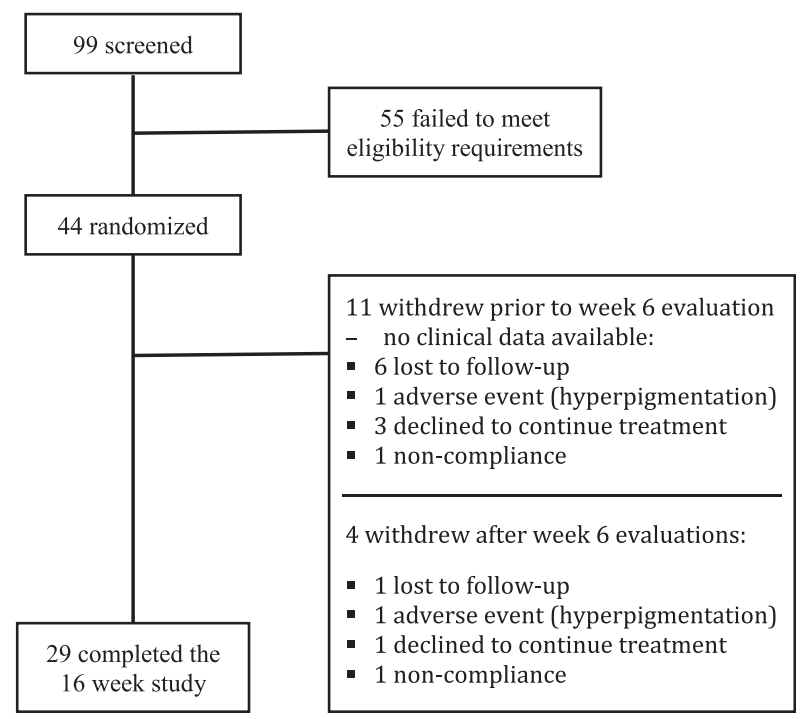

Figure 1 Trial profile.

There were also no statistically significant changes from baseline in lesion counts of any subtype of lesion when comparing treated and untreated skin on week 16 at the conclusion of the study, with one exception. Erythematous macules decreased in number bilaterally from baseline to week 16 , but the mean lesion decrease was statistically significantly more profound in treated skin $(-5.89)$ when compared with untreated skin $(-2.50)$, $P=0.04$. In general, there were mild decreases in most subtypes of lesions (papules, pustules, open, and closed comedones) at week 16 when compared with baseline. However, these changes occurred bilaterally on average such that there were no statistically significant differences in lesion counts between treated and untreated control skin $(P>0.05)$. One exception to the general trend toward bilateral improvement was a very mild increase in cyst counts bilaterally, but the baseline and week 16 mean cyst counts were very low (less than 1 per side on average) and this mild and statistically nonsignificant increase was not clinically relevant. Summary statistics of counts of all lesion subtypes are presented in Table 1.

Global acne severity scores decreased from baseline to week 16 bilaterally. The improvement in mean Leeds scores was more pronounced in treated skin $(-1.07)$ than untreated skin $(-0.52)$ and this difference was statistically significant $(P=0.01)$.

Additional statistical analyses were carried out to attempt to define and characterize subgroups of patients who did respond to the therapy. Responders were considered to be those who experienced at least a $25 \%$ decrease in lesion counts in treated skin when 
Photodynamic therapy for acne $-J$ S Orringer et al.

Table 1 Summary statistics of counts of all lesion subtypes at baseline, week 10, and week 16 in treated and untreated skin

\begin{tabular}{|c|c|c|c|c|c|}
\hline & & Baseline $(95 \% \mathrm{Cl})$ & Week $10(95 \% \mathrm{Cl})$ & $\Delta(95 \% \mathrm{Cl})$ & $P$-value \\
\hline \multirow[t]{2}{*}{ Papules } & Treated & $13.50(9.95-17.05)$ & $8.87(6.98-10.75)$ & $-4.63(-7.43$ to -1.83$)$ & 0.01 \\
\hline & Untreated & $13.57(10.59-16.55)$ & $13.43(10.17-16.70)$ & $-0.13(-2.83$ to 2.57$)$ & \\
\hline \multirow[t]{2}{*}{ Pustules } & Treated & $6.40(2.31-10.49)$ & $4.60(2.40-6.80)$ & $-1.80(-5.05$ to 1.45$)$ & 0.43 \\
\hline & Untreated & $6.33(2.58-10.09)$ & $5.13(2.97-7.30)$ & $-1.20(-4.49$ to 2.09$)$ & \\
\hline \multirow[t]{2}{*}{ Cysts } & Treated & $0.50(0.09-0.91)$ & $0.60(0.26-0.94)$ & $0.10(-0.35$ to 0.55$)$ & 0.79 \\
\hline & Untreated & $0.53(0.14-0.92)$ & $0.60(0.29-0.91)$ & $0.07(-0.30$ to 0.43$)$ & \\
\hline \multirow[t]{2}{*}{ Closed comedones } & Treated & $28.93(21.71-36.15)$ & $23.87(17.86-29.87)$ & $-5.07(-10.53$ to 0.40$)$ & 0.1 \\
\hline & Untreated & $30.27(24.77-35.76)$ & $29.10(22.37-35.83)$ & $-1.17(-7.52$ to 5.19$)$ & \\
\hline \multirow[t]{2}{*}{ Open comedones } & Treated & $13.13(4.92-21.34)$ & $12.13(5.37-18.89)$ & $-1.00(-6.37$ to 4.37$)$ & 0.73 \\
\hline & Untreated & $14.20(5.29-23.11)$ & $13.57(5.77-21.37)$ & $-0.63(-6.01$ to 4.75$)$ & \\
\hline \multirow[t]{2}{*}{ Erythematous macules } & Treated & $23.72(13.76-33.68)$ & $21.84(11.22-32.46)$ & $-1.88(-7.73$ to 3.97$)$ & 0.02 \\
\hline & Untreated & $23.36(11.89-34.83)$ & $24.64(13.94-35.34)$ & $1.28(-4.66$ to 7.22$)$ & \\
\hline \multirow[t]{3}{*}{ Severity } & Treated & $3.63(2.91-4.36)$ & $2.73(2.03-3.44)$ & $-0.90(-1.30$ to -0.50$)$ & 0.003 \\
\hline & Untreated & $3.67(3.00-4.34)$ & $3.37(2.70-4.04)$ & $-0.30(-0.65$ to 0.05$)$ & \\
\hline & & Baseline $(95 \% \mathrm{Cl})$ & Week $16(95 \%$ Cl) & $\Delta(95 \% \mathrm{Cl})$ & $P$-value \\
\hline \multirow[t]{2}{*}{ Papules } & Treated & $13.31(9.76-16.86)$ & $11.52(7.93-15.10)$ & $-1.79(-5.98$ to 2.39$)$ & 0.62 \\
\hline & Untreated & $13.66(10.59-16.72)$ & $12.69(9.11-16.27)$ & $-0.97(-4.32$ to 2.39$)$ & \\
\hline \multirow[t]{2}{*}{ Pustules } & Treated & $6.45(2.21-10.69)$ & $3.90(1.89-5.91)$ & $-2.72(-6.65$ to 1.20$)$ & 0.85 \\
\hline & Untreated & $6.38(2.49-10.27)$ & $3.76(2.03-5.49)$ & $-2.62(-6.25$ to 1.01$)$ & \\
\hline \multirow[t]{2}{*}{ Cysts } & Treated & $0.45(0.08-0.82)$ & $0.83(0.31-1.34)$ & $0.38(-0.20$ to 0.96$)$ & 0.49 \\
\hline & Untreated & $0.62(0.18-1.06)$ & $0.86(0.42-1.31)$ & $0.24(-0.33$ to 0.82$)$ & \\
\hline \multirow[t]{2}{*}{ Closed comedones } & Treated & $29.24(21.80-36.68)$ & $22.28(17.22-27.33)$ & $-6.97(-13.30$ to -0.63$)$ & 0.21 \\
\hline & Untreated & $29.38(23.48-35.28)$ & $25.31(20.02-30.60)$ & $-4.07(-9.12$ to 0.98$)$ & \\
\hline \multirow[t]{2}{*}{ Open comedones } & Treated & $15.00(5.95-24.05)$ & $10.21(5.09-15.33)$ & $-4.79(-11.62$ to 2.04$)$ & 0.27 \\
\hline & Untreated & $16.07(6.55-25.59)$ & $9.28(3.84-14.71)$ & $-6.79(-13.88$ to 0.29$)$ & \\
\hline \multirow[t]{2}{*}{ Erythematous macules } & Treated & $22.29(13.07-31.50)$ & $16.39(10.27-22.52)$ & $-5.89(-12.12$ to 0.34$)$ & 0.04 \\
\hline & Untreated & $21.71(11.21-32.22)$ & $19.21(11.82-26.61)$ & $-2.50(-8.85$ to 3.85$)$ & \\
\hline \multirow[t]{2}{*}{ Severity } & Treated & $3.63(2.88-4.38)$ & $2.56(2.7-3.04)$ & $-1.07(-1.69$ to -0.45$)$ & 0.01 \\
\hline & Untreated & $3.59(2.94-4.25)$ & $3.07(2.52-3.62)$ & $-0.52(-1.07$ to 0.04$)$ & \\
\hline
\end{tabular}

compared with baseline and who had at least 25\% fewer lesions on the treated side of the face when compared with untreated skin after completion of the final treatment session at week 6 . When defined in this way, a total of $8(18 \%)$ of patients were considered to be responders to the treatment. Further analysis was performed to determine whether patients with inflammatory or noninflammatory lesions were more likely to respond to the treatment. In this analysis, patients were considered responders if their inflammatory lesion counts (papules + pustules + cysts) were found to decrease by at least $25 \%$ as compared with baseline in treated skin and those total counts were found to be at least $25 \%$ lower than inflammatory lesion counts in untreated skin. Thirteen $(30 \%)$ patients were deemed responders in this way. A similar analysis was performed examining noninflammatory lesion counts (open comedones + closed comedones). Here, only three $(7 \%)$ patients were found to have responded to the PDT as defined above.

The treatments were generally well tolerated by patients and adverse events were mild and few in number. Two patients experienced mild peeling on the treated sides of their faces, two patients experienced transient postinflammatory hyperpigmentation in treated skin, and one patient developed a small blister on the day after treatment. All of these side effects resolved without permanent sequelae.

\section{Discussion}

Despite the availability of numerous effective medical therapies for acne vulgaris, issues of safety, compliance, and less than ideal efficacy help drive the search for alternative treatments for this exceedingly common clinical problem. There has been a long history of the use of light-based treatments for acne extending back decades wherein ultraviolet light was used to combat this condition. In recent years, the use of lasers and other light-based devices in the treatment of a wide variety of dermatologic disorders has been espoused. The boom in the use of these devices for esthetic applications has helped bring about renewed interest in developing effective light-based therapies for acne. 
Prior research has suggested a potential role for infrared and/or visible light lasers and light sources in the treatment of acne, yet most of this published work is in the form of small pilot studies without a randomized, controlled design. Unfortunately, in some cases, initial enthusiasm for a given treatment based on preliminary or pilot study results has led to later disappointment when larger, more rigorously designed clinical trials have been reported. Indeed, our own work in this area has previously demonstrated generally sub-optimal efficacy with the use of either infrared or visible light lasers for this indication. ${ }^{11,18}$

The use of topical photosensitizers to potentially enhance the effects of light-based therapies has proved to be a valuable strategy in the treatment of clinical photoaging. ${ }^{19-21}$ Others have also reported on the successful use of PDT for acne. Hongcharu and colleagues demonstrated significant improvement in back acne among patients treated with aminolevulinic acid and a noncoherent red light source, but the applicability of the treatment to many acne patients was questioned because of associated side effects such as hyperpigmentation and crusting. ${ }^{14}$ More recently, Horfelt et al. demonstrated a 54\% decrease in inflammatory acne lesions in treated skin when compared with a 20\% decrease in such lesions in control skin following a splitface clinical trial of PDT using methyl aminolevulinic acid and a noncoherent red light source. ${ }^{22}$ Similarly, the use of ALA and a broadband light source reportedly significantly decreased inflammatory lesion counts in a small uncontrolled clinical trial, but those authors noted that optimal treatment conditions and parameters have yet to be determined. ${ }^{23}$

There has been much speculation as to mechanistically how PDT might act to clinically improve acne. In vitro work involving a blue light source as reported by Ashkenazi and colleagues suggested that PDT might decrease counts of Propionibacterium acnes. ${ }^{24}$ Others have suggested that direct injury to sebaceous glands might underlie the clinical benefits of such treatment. ${ }^{6}$ Yet other researchers have failed to demonstrate an impact of PDT on either $P$. acnes counts or sebum production - results that clearly call into question the potential mechanisms involved. ${ }^{25}$ We found it to be of note that the effect of the treatment in our study appeared to be more profound on inflammatory lesions as opposed to noninflammatory lesions. If the mechanism of action truly involves an effect on bacterial counts or sebaceous gland structure and function, we would have expected to see equivalent improvement across all subtypes of acne lesions and this was clearly not the case. One might argue that the red color of the inflammatory lesions more efficiently absorbed the laser light than did the noninflammatory lesions. This might serve to make the treatment relatively more effective for inflammatory lesions, whatever the mechanism involved might be, but this is purely speculative. In any case, our finding that more patients responded with respect to their inflammatory lesion counts is in keeping with the findings of a recent PDT consensus panel.

It was also noteworthy that erythematous macules cleared to a statistically significant degree in treated skin. Again, the oxygenated hemoglobin within the dilated vessels that produced the erythema in these lesions might have served as a chromophore for the pulsed dye laser light. This would account for the preferential clearing of these remnants of previously inflammatory lesions in skin treated with PDT. Another notable finding was the fact that overall mean inflammatory papule counts were found to improve in treated skin by week 10, but that this positive effect was no longer evident by week 16 when counts from all patients were considered. It is possible that temporary decreases in bacterial counts may have contributed to this phenomenon with repopulation of the bacteria accounting for the transient nature of the improvement. In any case, such a finding argues for the possible need for ongoing maintenance therapy to sustain any clinical benefits of the treatment. In addition, interesting in our results was the finding that patients' mean global acne severity scores did decline more substantially in treated skin than in control skin. While individual lesion counts are perhaps the gold standard endpoint for measuring clinical efficacy, the fact that global severity ratings using the Leeds scale did significantly improve with this treatment does suggest potential benefit of this treatment regimen.

This study was limited in that it defines outcomes for one specific, albeit clinically popular, PDT regimen. It is possible that by varying a number of treatment parameters (including the light source used, the photosensitizer employed and its contact time, etc.), more significant clinical improvements may be achievable. In addition, the early withdrawal rate in this clinical trial acts to decrease the sample size and thus the statistical power. However, the number of participants in this study compares favorably to most reported clinical trials of light-based therapies for acne. Furthermore, the current results only speak to the level of efficacy of this treatment protocol among our specific patient sample group. It is possible that this form of PDT might prove to be either more or less 
effective among patients with relatively milder or more severe acne at baseline.

Previous research involving a similar ALA and pulsed dye laser-based approach using treatment parameters that were nearly identical to those employed in the current study demonstrated complete clearance of all acne lesions among all 14 patients treated in this fashion. ${ }^{16}$ However, subjects in that study were also prescribed other concurrent anti-acne medications making interpretation of the findings difficult. Unfortunately, we were not able to duplicate these previously reported very positive results and, in fact, only a minority of our patients responded to the treatment in a significant fashion. Further confounding our efforts to assess the validity of this treatment approach was the fact that, even among responders as defined above, we were unable to define patient characteristics (age, baseline acne severity, gender, etc.) that were predictive of treatment outcomes. Our results suggest that, with the specific treatment protocol employed in this study, results with respect to acne improvement can be expected to be rather modest and inconsistent. However, the fact that some patients did improve with the current regimen allows for the possibility that future work examining dose response curves, optimization of light source, definition of ideal photosensitizer application times and conditions, and a number of other variables might one day make PDT for acne an important therapeutic tool. For now, our data suggest that PDT using the pulsed dye laser is a second or third line treatment option to be considered for patients with inflammatory acne who have not responded to traditional medical therapies.

\section{Acknowledgments}

We wish to thank Jennifer Keith for her assistance with patient recruitment and study coordination and Laura Van Goor for her work on producing the tables and figures for this manuscript. We would also like to thank Trivellore Raghunathan, Maria Larkina, and Miquelle Milavec for their assistance with data analysis. Funding Sources: This study was supported by the University of Michigan Department of Dermatology Laser Research Fund.

\section{References}

1 Webster G. Acne vulgaris. BMJ 2002; 325: 475-9.

2 Layton A. Psychosocial aspects of acne vulgaris. J Cutan Med Surg 1998; 2(Suppl. 3): 14-8.
3 Cunliffe W. Acne and unemployment. Br J Dermatol 1986; 115: 386 .

4 Papageorgiou P, Katsambas A, Chu A. Phototherapy with blue $(415 \mathrm{~nm})$ and red $(660 \mathrm{~nm})$ light in the treatment of acne vulgaris. Br J Dermatol 2000; 142: 973-8.

5 Stillman S, Geen S, Harth Y, Shalita A. High intensity narrow band blue light is effective in the treatment of acne vulgaris: an in vitro and in vivo study. J Eur Acad Dermatol Venereol 2000; 14(Suppl. 1): 47.

6 Paithankar D, Ross E, Saleh B, Blair M, Graham B. Acne treatment with a $1,450 \mathrm{~nm}$ wavelength laser and cryogen spray cooling. Lasers Surg Med 2002; 31: 106-14.

7 Ross V. Optical treatments for acne. Dermatol Ther 2005; 18: $253-66$.

8 Mariwalla K, Rohrer T. Use of lasers and light-based therapies for treatment of acne vulgaris. Lasers Surg Med 2005; 37: 333-42.

9 Baugh W, Kucaba W. Nonablative phototherapy for acne vulgaris using the KTP $532 \mathrm{~nm}$ laser. Dermatol Surg 2005; 31: 1290-6.

10 Seaton E, Charakida A, Mouser P, Grace I, Clement RM, Chu AC. Pulsed-dye laser treatment for inflammatory acne vulgaris: randomised controlled trial. Lancet 2003; 362: 1347-52.

11 Orringer J, Kang S, Hamilton T, Schumacher W, Cho S, Hammerberg C, Fisher GJ, Karimipour DJ, Johnson TM, Voorhees JJ. Treatment of acne vulgaris with a pulsed dye laser. JAMA 2004; 291: 2834-9.

12 Wiegell S, Wulf H. Photodynamic therapy of acne vulgaris using methyl aminolaevulinate: a blinded, randomized, controlled trial. Br J Dermatol 2006; 154: 969-76.

13 Akaraphanth R, Kanjanawanitchkul W, Gritiyarangsan P. Efficacy of ALA-PDT vs. blue light in the treatment of acne. Photodermatol Photoimmunol Photomed 2007; 23: 186-90.

14 Hongcharu W, Taylor C, Chang Y, Aghassi D, Suthamjariya K, Anderson R. Topical ALA-photodynamic therapy for the treatment of acne vulgaris. J Invest Dermatol 2000; 115: 183-92.

15 Hong S, Lee M. Topical aminolevulinic acid-photodynamic therapy for the treatment of acne vulgaris. Photodermatol Photoimmunol Photomed 2005; 21: 322-5.

16 Alexiades-Armenakas M. Long-pulsed dye laser-mediated photodynamic therapy combined with topical therapy for mild to severe comedonal, inflammatory, or cystic acne. J Drug Dermatol 2006; 5: 45-55.

17 O’Brien S, Lewis J, Cunliffe W. The Leeds revised acne grading system. J Dermatol Treat 1998; 9: 215-20.

18 Orringer J, Kang S, Maier L, Johnson TM, Sachs DL, Karimipour DJ, Helfrich YR, Hamilton T, Voorhees JJ. A randomized, controlled, split-face clinical trial of 1320-nm Nd:YAG laser therapy in the treatment of acne vulgaris. J Am Acad Dermatol 2007; 56: 432-8.

19 Orringer J, Hammerberg C, Hamilton T, Johnson TM, Kang S, Sachs DL, Fisher GJ, Voorhees JJ. Molecular effects of 
photodynamic therapy for photoaging. Arch Dermatol 2008; 144(10): 1296-302.

20 Dover JS, Bhatia AC, Stewart B, Arndt KA. Topical 5-aminolevulinic acid combined with intense pulsed light in the treatment of photoaging. Arch Dermatol 2005;

141: $1247-52$.

21 Touma DJ, Gilchrest BA. Topical photodynamic therapy: a new tool in cosmetic dermatology. Semin Cutan Med Surg 2003; 22: 124-30.

22 Horfelt C, Funk J, Frohm-Nilsson M, Wiegleb Edström D, Wennberg AM. Topical methyl aminolaevulinate photodynamic therapy for treatment of facial acne vulgaris: results of a randomized, controlled study. Br J Dermatol 2006; 155: 608-13.
23 Lee J, Kim S, Park K, Son S. Topical aminolaevulinic acid-photodynamic therapy for the treatment of mild to moderate inflammatory acne. Ann Dermatol 2007; 19: 55-9.

24 Ashkenazi H, Malik Z, Harth Y, Nitzan Y. Eradication of Propionibacterium acnes by its endogenic porphyrins after illumination with high intensity blue light. FEMS Iттиnol Med Microbiol 2002; 35: 17-24.

25 Pollock B, Turner D, Stringer M, Bojar RA, Goulden V, Stables GI, Cunliffe WJ. Topical aminolaevulinic acidphotodynamic therapy for the treatment of acne vulgaris: a study of clinical efficacy and mechanism of action. $\mathrm{Br} \mathrm{J}$ Dermatol 2004; 151: 616-22. 